\title{
A Game-Theoretic Model of Disclosure-Donation Interactions in the Market for Charitable Contributions
}

\author{
Gregory D. Saxton \& Jun Zhuang
}

The information organizations choose to disclose is a strategic consideration. The "success" of strategic disclosure is contingent in turn on the target audience's values and preferences. The disclosure of and reaction to organizational information thus involves a strategic interaction between the producer and consumer of the information, yet prior communication research has focused on only one side or the other of this equation. In response, we examine organization-public interactions in a domain with key, measurable outcomes - the charitable donations marketplace - and propose a game-theoretic explanation of organizational disclosures and individual donations. The study specifically emphasizes two core donor preferences - the desire for impact and the desire for publicity - with donors choosing between organizations based on how well the organization satisfies these preferences. Organizations' optimal level of disclosure, in turn, depends on their own "type" and the types of donors they attract. This study recommends organizations think of their disclosure and efficiency-related decisions as conveying valuable, yet distinct, information to two ideal-types of prospective donors: the impact maximizer and the publicity maximizer. Mapping and targeting the "right" mix of donors is a key managerial decision that ultimately impacts both the organization's financial outcomes and donors' satisfaction with the donation experience.

\begin{abstract}
Keywords: Organizational Communication; Voluntary Disclosure; Nonprofit Organizations; Donations; Prosocial Behavior; Game Theory; Organization-Public Relations
\end{abstract}

\footnotetext{
Gregory D. Saxton is an Assistant Professor in the Department of Communication at the University at Buffalo, State University of New York. His interests are in new media and organizational communication, particularly with regard to nonprofit organizations. Jun Zhuang is an Assistant Professor in the Department of Industrial and Systems Engineering at the University at Buffalo, SUNY. Dr. Zhuang's long-term research goal is to integrate operations research, game theory, and decision analysis to improve mitigation, preparedness, response, and recovery for natural and man-made disasters. Correspondence to: Gregory D. Saxton, Department of Communication, University at Buffalo, SUNY, 331 Baldy Hall, Buffalo, NY 14260, USA. E-mail: gdsaxton@ buffalo.edu
} 
Information disclosure is a communication tool that individuals and organizations alike employ to influence decision making. This is keenly evident in the market for charitable contributions, where nonprofit organizations try to sway potential donors through the strategic release of financial, performance, and fundraising-related data intended to not only boost accountability and public trust but also attract greater donations (Gandía, 2011; Saxton \& Guo, 2011). To better comprehend such processes involving both the determinants and behavioral outcomes of communication decisions, we need a theoretical approach that integrates the strategic, interactive nature of decision making undertaken by both producers and consumers of information.

Communication studies to date have generally focused exclusively on either the source or the receiver of this communication process. To remedy this, we propose a game-theoretic explanation of disclosure-donation interactions. Such an approach explicitly incorporates the decision making undertaken by both the donor and the discloser in the fundraising arena. Our model also recognizes that payoffs associated with strategically disclosed fundraising information are contingent on individual donors' values and preferences: Donors react differently based on the extent to which they are motivated by altruism, vested interests, fairness, social impact, publicityseeking, or the "warm glow" from donating (e.g., Anker, Feeley, \& Kim, 2010; Bergstrom, Blume, \& Varian, 1986; Sargeant, 2001). By incorporating multiple forms of donor utility (satisfaction), the model strives to explain the communicative and prosocial outcomes generated by donor-organization interactions using a realistic range of organizational and donor objectives.

To facilitate the presentation of the model, in the next section we present some brief background information on the market for charitable contributions, concentrating on the role of disclosure. We then review the relevant literature on organizational disclosure and the drivers of individual donations before presenting a summary of our game theory method and analysis plan. The presentation of our game theory model and solution follows. We then analyze how optimal levels of donations and disclosure are influenced by changes in each of the key variables, and discuss the testable propositions generated by the theoretical model. Last, given our belief that the model we develop has applications for organizational and interpersonal communication scholars interested in the interplay of communication and behavior, we end with a discussion of the larger theoretical implications as well as concrete practical applications of our study.

\section{Background: Information in the Market for Charitable Contributions}

Though they go by many names-including not-for-profits, charities, and nongovernmental organizations (NGOs) - nonprofit organizations throughout the world have three things in common: (1) they are voluntary and self-governing, (2) they must not distribute profits, and (3) they serve the public as well as the common goals of their members (for an overview, see Lewis, 2005). In democratic countries such as the United States, nonprofits play a vital role in sustaining civil society by delivering 
much-needed services and programs in areas such as education, the arts, sports, the environment, humanitarian aid, and health care. This work depends heavily on donations from private citizens and corporations; in the United States alone, annual donations total roughly $\$ 300$ billion (Blackwood, Wing, \& Pollak, 2008). Donations are a vital revenue source, and the competition can be intense.

In many ways, it is thus reasonable to refer to the nonprofit sector as a "market," with organizations competing to raise funds by securing "investors" who help the organization meet its social mission by making a donation. In fact, the marketization of the charitable sector has intensified over the past two decades, as governmental, private, and corporate contributors have all come to emphasize accountability, return on investment, and verifiable results over good intentions (e.g., Behn, 2005). Over the same period, the diffusion of Web sites, blogs, and social media has turned the Web into a crucial informational channel (Kent, Taylor, \& White, 2003; Lovejoy \& Saxton, 2012; Saxton \& Guo, 2011), to which most prospective donors can turn for information on the organization's efficiency, effectiveness, fundraising activities, and governance.

Both trends have increased donors' demand for information and, in the process, rendered organizational disclosure even more important. Not only does the direct provision of information help organizations better inform prospective donors but, in the aggregate, by mitigating the considerable information asymmetries that exist between donor and charity (e.g., Gordon, Knock, \& Neely, 2009), disclosures help create a more efficient market. Disclosure is hence increasingly seen as a vital financial reporting, accountability, and governance tool (e.g., Saxton \& Guo, 2011).

\section{Example: Aftermath of 2011 Japanese Earthquake and Tsunami}

A brief example will shed light on the nature of the charitable contributions market and introduce our theoretical "problem." Specifically, let us consider the humanitarian response to the March 2011 earthquake and tsunami in Japan. Reflecting the market transformations noted earlier, the Web served as a powerful donations tool as well as a vehicle for rapid information aggregation and dissemination. Total donations were substantial: in the first 5 days, the American Red Cross alone had received \$34 million to support its relief efforts (Strom, 2011). Given the amount of donations made and the scale of the rebuilding efforts, donors should get what they think they are "paying" for.

The Donor's Problem. The prospective donor seeks to make a contribution to support the tsunami-relief efforts. Numerous charities were soliciting donations for relief services - the Red Cross, Oxfam, Mercy Corps, Project HOPE, Americares, Samaritan's Purse, Kiwanis International, Doctors Without Borders, Lion's Club International, and many others - so the fundamental question for the donor is, "How can I decide where to send my money, and how much should I give?" The typical donor has a clear aim to maximize the humanitarian impact from his or her donation, and most donors would also appreciate some recognition acknowledging that support (Sargeant, 2001). The question is, given the substantial asymmetry of 
information between the charity and the donor concerning each organization's relative effectiveness, how can the donor choose?

The Nonprofit's Problem. An organization can differentiate itself through disclosure. By selectively disclosing information regarding fundraising activities, stakeholder relations, internal actions, and social and financial performance, an organization can gain market share and boost charitable contributions (Weisbrod \& Dominguez, 1986). However, too much disclosure may result in "information overload" and alienate donors and other stakeholders. Moreover, poorly governed organizations will not do well by disclosing, and any organization could mistakenly release materialssuch as a list of donors or insights into organizational strategy - that become valuable to competitors (Healy \& Palepu, 2001). Deciding how much to disclose is thus a key managerial decision.

\section{Disclosure and Donations in the Literature}

The example in the previous section highlights the strategic considerations of two key actors: the donor, who is the receiver of the information, and the organization, which is the source. Our model addresses these considerations by describing optimal levels of disclosure and donations based on the nonprofit's level of effectiveness and the donor's preferences for publicity and social impact. Before presenting the model, we first lay out how it builds on existing multidisciplinary research on the motivations for disclosure and donations.

\section{Motivations for Disclosure}

Disclosure is a common topic of communication research. Interpersonal communication scholars have devoted considerable attention to the issue of self-disclosure (e.g., Myers \& Johnson, 2004; Stefanone \& Jang, 2008). In communication and psychology, such concepts as "impression management" have been used to examine the uses of interpersonal disclosure (see, for instance, Hancock \& Toma, 2009). However, organizational-level analyses have not been as common. Studies have been done that attempt to categorize the types of disclosure, such as "official" versus "unofficial" disclosure (Bishop, 2006), and it has been a minor element of various other studies, including at least one study on "information adequacy" within organizations (Rosenfeld, Richman, \& May, 2004). A handful of studies have begun to explore disclosure as a key component of organization-public relationships (e.g., Huang, 2001), with several either implicitly or explicitly addressing strategic disclosure in organization-public relations. Notably, Arpan and Roskos-Ewoldsen (2005) examine "proactive" disclosure during organizational crises, whereas Sweetser (2010) examines the effects of nondisclosure on public relations.

Collectively, the communication literature-when it does deal with organizations - strongly implies a strategic aspect to the disclosure of information. However, it rarely deals with the "public" side of the equation. Only one published study (Rawlins, 2009) seriously attempts to measure the effects - namely, the public's 
response - to organizational disclosure. To date, interactive, strategic approaches have received limited attention in communication research. Still, these studies have been useful in moving the literature to examine new domains of strategic organizational communication. In this paper we devote ourselves to moving the literature down the same path by explicitly incorporating the interactive nature of communication-related decision-making processes.

Fundraising-specific disclosure and the publicity-maximizing donor. The economics literature is the primary locus of game-theoretic research on organizational disclosure (Zhuang \& Bier, 2010, 2011). By far the largest group of nonprofit-focused game theory studies are those that build on Bergstrom et al.'s (1986) groundbreaking study on the private provision of public goods. Dozens of studies have built on their model by incorporating the effects of organizational disclosure on donations (Clark, 2002; Romano \& Yildirim, 2001). However, this research has only focused on a certain domain of disclosure: fundraising disclosure. This refers to the release of details on the organization's fundraising campaigns, including the number of potential donors, contributors' names, and the amounts they have given. It covers all announcements - on any medium - related to specific donations or the progress of a fundraising campaign, such as the naming of buildings, donor names on commemorative bricks, announcement of donors on a crowdfunding site, a university's public recognition of a large donation, or a small organization's listing of individual contributions on a website or in an online or printed annual report. This literature effectively suggests that such disclosures can sway the behavior of subsequent donors. However, in examining only fundraising disclosures, the economics-based "public goods" literature has focused on donors whose utility derives chiefly from publicity and social pressures and praise flowing from fundraising-related publicity. We extend this research by incorporating additional motivations for donating.

\section{Motivations for Donating: Organizational Effectiveness and the Impact-Maximizing Donor}

In contrast to the economics literature, the conceptual and empirical literature in communication, nonprofit studies, accounting, political science, and social work, among others, has overwhelmingly posited a different motivation-that donors seek to maximize their impact, which they accomplish by donating to efficient and effective organizations. Impact-seeking donors could be affected by disclosures about previous donations, such as seeing higher levels of donations to an organization as an indirect proxy for organizational quality. Similarly, the donor might take the absence of disclosure as a signal the organization has something to hide. However, "impactmaximizing" donors are not so much interested in fundraising disclosure, or who has already donated and how much, but in the organization's effectiveness - in how well it is fulfilling its stated social mission (e.g., Lewis, 2005; Moore, 2000; Saxton \& Guo, 2011; Saxton, Neely, \& Guo, 2009). 
Marketing and communication research has studied broadly similar issues. For example, health communication scholars have found messages encouraging "selfefficacy" among target audiences are an effective means for boosting blood and organ donations (Anker et al., 2010). In the charitable contributions arena, scholars have found donation decisions are influenced by organizational performance, impact, and the perception of benefits (Sargeant, Ford, \& West, 2006). These types of factors may all be directly influenced by the organization's levels of effectiveness.

However, it is notoriously difficult to develop valid, reliable, and comparable measures of performance in the nonprofit sector (Parsons, 2003). For this reason, efficiency ratios are widely used as imperfect proxies for effectiveness. For instance, organizations are often compared using the program spending ratio, which taps the proportion of organizational spending devoted to programs as opposed to fundraising or administration. If one organization devotes only $70 \%$ of expenses to programs whereas another devotes $90 \%$, the latter is more efficient and, generally speaking, is likely more effective. Accordingly, it is this ratio that is often highlighted on third-party charity evaluation Web sites such as Charity Navigator or Guidestar (Gordon et al., 2009).

Weighing in on this issue is a nascent body of empirical research in nonprofit studies, political science, and accounting on the determinants of charitable giving. This literature has consistently found that donors are influenced by financial efficiency ratios (see Parsons, 2003, for an overview), with the few studies that have examined nonfinancial measures of effectiveness finding mixed evidence (Reck, 2001). Except for Reinhardt (2009), this line of research has not utilized formal gametheoretic models, nor has it looked at the effects of fundraising disclosures. Instead, such research has been limited to empirically examining the donor relevance of broad, widely available measures of organizational efficiency and effectiveness.

\section{Summary of Literature and Theoretical Expectations}

Our current understanding of organizational disclosure in the nonprofit context is not deep. Prior game-theoretic studies have focused on the disclosure of details related to fundraising campaigns and have emphasized donors with "publicitymaximizing" utility functions, whereas empirical studies have focused on the effects of publicly available financial efficiency ratios on individual and aggregate levels of donations. Furthermore, previous research has not formally modeled the interactions between organizational disclosures and charitable contributions by donors with "impact-maximizing” utility functions.

Organizational communication studies, meanwhile, have implicitly identified the strategic decision making that underlies organizational disclosure, but have yet to formally model the strategic interplay between informational producer and consumer. In effect, in attempting to understand the donation/disclosure nexus, existing communication theory has focused on only one side or the other of this equation. Such an approach can inform our thinking but cannot provide a complete explanation of this decision-making process. The key to understanding this 
interaction is the recognition that donors will react strategically to disclosure based on their individual preferences, and that organizations will attempt to incorporate these donor preferences beforehand into their disclosure decision making. It is in this sense that the decisions to release information and to donate money are interactive processes.

In brief, existing game-theoretic and empirical studies in economics and nonprofit studies have generally ignored one or the other of the two core facets of donor preferences, and communication studies have not incorporated interactive decision making into their theoretical models. To help propel the organizational communication and nonprofit disclosure literatures further, we need an approach that formalizes the interactive decision making undertaken by the producer and consumer of organizational information. We need an approach that recognizes certain donors' desire for publicity. And we need an approach that accounts for donors' desire to have a meaningful impact through their charitable contributions. We address these needs through a game-theoretic account of the disclosure-donations nexus that connects the two primary forms of donor utility with the organizational release of information related to fundraising campaigns.

\section{Method}

In this paper we employ a formal modeling approach, specifically game theory, as a theory-building tool. There are two general approaches to game theory studies. For previously existing game theory models, the known equilibrium solutions can be tested (or validated) via empirical research or laboratory experiments in order to compare the theoretical equilibrium strategy with observed behavior (e.g., Carpenter, Connolly, \& Myers, 2008). For new models such as ours, however, the approach involves formally setting up a game theory model and then solving the model. We then assess the implications of our model through a series of sensitivity analyses that show how expected donations and disclosures vary with changes in the model parameters. Given the novelty of this approach to studying communication-related issues, in this section we present a brief overview of the nature, benefits, and uses of game-theoretic approaches to social scientific problems. We then present our analysis plan. In the subsequent section we present the details of our model, the model solution, and the sensitivity analyses.

\section{Benefits and Uses of Game Theory}

Game theory is a theory of interactive decision making. Game-theoretic approaches are thus ideally suited to studying situations involving strategic interactions between social actors (for an overview, see Morrow, 1994; Zagare, 1984). By "game," game theorists "are referring to any social situation involving two or more actors (players) in which the interests of the players are interconnected or interdependent" (Zagare, 1984 , p. 7). In specifying a game, the theorist lays out who the key players are, the choices available to the players, the relationship between choices and consequences, 
and how players evaluate different outcomes. In effect, the theorist elaborates a formal structure in which players' interdependent decision making takes place. In Morrow's words $(1994$, p. 1), game theory thus "provides a way to formalize social structures and examine the effects of structure on individual decisions." These "individual" decisions can be modeled at any level of analysis; in our case, we focus on the interactive decision-making processes of organizations and donors.

A primary benefit of a game-theoretic approach is the rigor and precision required in any formal argument. Assumptions and assertions about the characteristics of a situation must be formally, mathematically written down, forcing a discipline on our intuition (Morrow, 1994). Moreover, a game-theoretic model allows one to see precisely why and how conclusions follow from the assumptions made. In itself, game theory does not tell us whether a particular theory is an accurate reflection of reality; instead, it presents a set of precise and testable hypotheses regarding specific behaviors that are directly inferable from the theory (Morrow, 1994).

Often, this analysis leads to unexpected insights and conclusions that go beyond initial intuition. As Fink (1993, p. 5) argues, "[a] mathematical model is capable of generating surprising, or nonobvious, or fertile implications which shed light on the theory that generated it, and which wouldn't be seen without the extension in reasoning that the mathematics provides." A key goal is that, through this rigorous and explicit formal modeling, scholars "generate streams of research that lead to areas never anticipated by the original work" (Morrow, 1994, p. 2).

\section{Analysis Plan}

After formally specifying the model, we solve it. The solution will yield two equations, which describe the equilibrium, or optimal, levels of disclosure and donations, respectively, given the organization's and donor's values for key parameters in the model (see the Results section for complete details). These solutions to game theory models are generally referred to as equilibrium solutions, in the sense that neither player has an incentive to alter his or her behavior-each player's decision produces the most favorable outcome for that player, taking into account the other player's action.

After finding the equilibrium solution, the implications of the solution will be explored using a technique known as sensitivity analysis (see, among others, Fiacco \& Ishizuka, 1990). Sensitivity analysis is the process of systematically changing values of the input (independent) variables in a model to see the effects of those changes on the output (dependent) variables. In our case, the sensitivity analysis affords a way of studying how the two key outputs - optimal donations and disclosures - vary in response to changes in the level of organizational efficiency or donor preferences for publicity or impact, holding the other model variables constant at some chosen value. In addition to facilitating an examination of the nature of the relationship between the independent and dependent variables, sensitivity analyses are key to identifying unexpected relationships, nonlinear relationships, and minimum and maximum 
values. A key goal is to utilize these analyses to generate unexpected insights and posit novel hypotheses that can be empirically tested in subsequent research.

\section{The Model}

The aim of formal modeling is to capture "the essence of the problem as simply as possible" (Morrow, 1994, p. 312). Accordingly, to help build a foundation for our theoretical arguments, we restrict our analysis to the case of one organization, one donor. Though at first glance simplistic, the goal is to tap into what Fink (1993, p. 5) calls the "wonderful paradox" of successful mathematical models of theories: "Great models, generated from apparently simple theories, lead to insights we would not have without the models." In this way, we make several "simplifying" assumptions in setting up the model. We assume a single-service organization that relies on donations for a large proportion of its revenues. In line with arguments made earlier, we maintain that organizations can increase their chances of obtaining donations by delivering organizational communication strategies that tap into donors' "publicity-maximizing" preferences. The organization's executive director must decide whether to voluntarily disclose fundraising-related information, such as who has already donated, how much, and when. The organization seeks to maximize net charitable contributions (Weisbrod \& Dominguez, 1986), and the release of detailed fundraising campaign information could positively or negatively impact donations depending on donors' relative preferences for publicity and impact.

In effect, there are two key players, one nonprofit organization and one donor, with the nonprofit organization first deciding whether to disclose or to not disclose, and the donor then deciding whether to provide funding to the organization (see Figure 1). We assume the nonprofit moves first because, in reality, a donor is able to observe an organization's disclosure effort before making donations. Players are assumed to be "rational," or goal-oriented, in that each player will act in a way that delivers the most preferred outcome from the available options (Zagare, 1984); and we assume each player knows the general payoffs and strategies available to the other player. These initial assumptions are all in line with existing game-theoretic models, such as Axelrod's (1984) highly influential book The Evolution of Cooperation, which led to key insights into cooperation using a simplistic two-player model. These assumptions make the model tractable but still provide useful insights.

We then define the parameters (variables) of our model as shown in Table 1. At the heart of the model are two key equations, the utility functions, that summarize the payoffs for the two players. In line with the goal of maximizing net levels of charitable contributions, the nonprofit's utility is a function of the amount of donations it receives $(x)$ less the costs associated with fundraising disclosure $(y)$, defined as earlier as announcements related to the progress of a fundraising campaign, such as who has donated when and how much. In particular, we employ a quadratic form of 


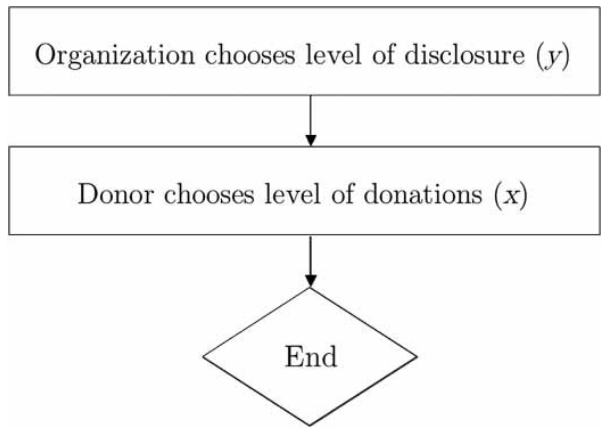

Figure 1. Sequence of moves for one organization and one donor.

fundraising disclosure costs $\left(y^{2}\right)$, which serves to approximate a budget constraint. The organization's utility is thus given by,

$$
U_{N}(x, y)=x-y^{2}
$$

The quadratic term $\left(y^{2}\right)$ reflects the assumption that costs increase with the level of fundraising disclosure. In short, the relationship between fundraising disclosure and organizational utility is initially positive; however, as disclosure costs continue to increase, it becomes increasingly harder to earn an additional net dollar in donations, to the point where the utility derived from disclosure eventually turns negative. In keeping with the assumption that both players seek to maximize utility, the quadratic term thus serves as a budget constraint that averts predictions of unrealistically high levels of disclosure expenditures.

Next, the second mover - the donor-wishes to maximize the societal impact as well as the amount of personal publicity he or she receives from making the donation, net of the loss accrued from making the donation. The donor's utility is thus modeled as a function of three elements. First, the payoff associated with the desire to achieve an impact is a product of the donor's level of donation $(x)$ to the organization, the level of organizational effectiveness $(\lambda)$, and the donor's impact preference $(\gamma)$. In line with the literature reviewed earlier, measures of organizational efficiency and effectiveness are deemed to be readily available. Second, the payoff associated with publicity is a product of the donor's publicity preference $(\alpha)$, the amount of the donation $(x)$, and the level of fundraising-related disclosure $(y)$. Third, the cost associated with making the donation is modeled as a function of the

Table 1 Model parameters

\begin{tabular}{lrl}
\hline Variable & \multicolumn{1}{c}{ Range } & \multicolumn{1}{c}{ Description } \\
\hline$x$ & $\geq 0$ & Amount of charitable contributions made by donor \\
$y$ & $\geq 0$ & Amount of fundraising disclosure made by organization \\
$\lambda$ & $0 \rightarrow 1$ & Efficiency/effectiveness of the organization \\
$\gamma$ & $\geq 0$ & Donor's preference for donating to effective organization \\
$\alpha$ & $-\infty \rightarrow \infty$ & Donor's preference for personal publicity \\
$\beta$ & $\geq 0$ & Donor's preference for retaining money \\
\hline
\end{tabular}


donation amount $(x)$ and the donor's "disutility" from giving away money or, differently put, preference for retaining money $(\beta)$. Specifically, we assume a quadratic donation cost $\left(x^{2}\right)$ for the donor, which, as in the organization's case, approximates a budget constraint. The donor's utility function is thus given by,

$$
U_{D}(x, y)=\underbrace{\gamma \lambda x}_{\text {impact }}+\underbrace{\alpha x y}_{\text {publicity }}-\underbrace{\beta x^{2}}_{\text {cost }}
$$

As shown in Equation 2, impact, personal publicity, and cost are the three elements driving the donor's utility, or overall payoff from the donation decision. This simple, yet informative model thus captures three key donor preferences, of which previous game theory models have been missing the first and arguably the most important.

\section{Results}

\section{Solution to the Model}

The solution to the type of game we have elaborated is known as a Nash equilibrium, where each player's decision produces the most favorable outcome for that player, taking into account the other player's action (for an overview of the concept, see Dixit, Skeath, \& Reiley, 2009). Each player's decision is thus what is known as a "best response" to the other player's strategy; neither has an incentive to make a unilateral change in strategy. In our case here, for the organization, the best response (optimal disclosure) will be the level of disclosure that maximizes the organization's payoff (net amount of donations), taking into account the donor's expected level of contributions; for the donor, the best response (optimal donations) will be the level of donations that maximizes his or her overall payoff, taking into account the observed level of organizational disclosure.

If we let $y^{*}$ be the organization's equilibrium (optimal) disclosure, and $x^{*}$ be the donor's equilibrium (optimal) donation, then as shown in the Appendix, when $\alpha \geq$ 0 , the Nash equilibrium solution to the model is:

$$
x^{*}=\left(\frac{4 \beta \gamma \lambda+\alpha^{2}}{8 \beta^{2}}\right) \text { and } y^{*}=\left(\frac{\alpha}{4 \beta}\right)
$$

Thus, the equilibrium solution represents a range of values (also known as a frontier), where any hypothetical donor's or organization's precise value for $x^{*}$ or $y^{*}$, respectively, depends on the specific values of the parameters $\alpha, \beta, \gamma$, and $\lambda$.

\section{Sensitivity Analyses}

In this section we conduct sensitivity analyses based on the information contained in these two equations. As noted earlier, sensitivity analysis allows us to isolate the effects of changes in one variable by holding the other variables in the model constant at some baseline value. Specifically, the focus is on how the outcome variablesdisclosure and donations - are affected by changes in the other model parameters. 
As shown earlier, optimal disclosure $\left(y^{*}\right)$ is a function of donor preferences for personal publicity $(\alpha)$ and retaining money $(\beta)$, whereas optimal donations $\left(x^{*}\right)$ is a function of the organization's level of efficiency/effectiveness $(\lambda)$ as well as donor preferences for publicity $(\alpha)$, impact $(\gamma)$, and money retention $(\beta)$. Now, each organization and donor may have different values, preferences, and characteristics, which would be reflected in variation in each of these model parameters. In the analyses that follow we are going to examine the effects of changes in each of these parameters in turn, holding the other parameters constant. This effectively allows us to answer such questions as, "What happens to optimal donations as values of organizational efficiency vary?" Collectively, the sensitivity analyses highlight the key effects of the independent variables on optimal levels of disclosure and donations.

For ease of exposition, the following analyses consider the measure of effectiveness to be the "program-spending ratio," the most well-known measure of organizational efficiency. Though there are crucial differences between efficiency and effectiveness, measures of organizational impact are notoriously difficult to develop and, as a result, as argued earlier, many organizations and donors alike rely instead on widely available efficiency ratios as an indirect but concrete, readily comparable, and "reliable" indicator of effectiveness. Specifically, let our empirical proxy for effectiveness $(\lambda)$ be the organization's ratio of program expenditures to total expenditures - such that a $\lambda$ value of 0 indicates a wholly inefficient organization, or one that devotes all of its expenses to fundraising and administrative expenses and nothing to programs; and a value of 1 indicates a wholly efficient organization, one that devotes all of its expenses to programs. For instance, a donor conducting a simple Web search would find program-spending ratios for the tsunami relief-effort organizations listed earlier that range from $41.9 \%$ for Kiwanis International to $99.0 \%$ for Americares. There is thus a broad range of efficiency levels even for reputable organizations such as these. The Charity Navigator site shows official US 501(c)(3) organizations with program-spending ratios as low as $2.2 \%$, and scam organizations would typically have $0 \%$ program expenses.

Figure $2 \mathrm{a}$ shows the sensitivity of optimal donations $\left(x^{*}\right)$ and disclosure $\left(y^{*}\right)$ to a change in the donor's publicity preference $(\alpha)$, holding all other parameters $(\beta, \gamma, \lambda)$ equal to 1 (an arbitrary but meaningful value indicating, respectively, positive preferences for money retention and impact and a high positive level of organizational efficiency). The figure shows that optimal disclosures increase linearly with the donor's publicity preference, whereas optimal donations see a nonlinear (convex) positive rate of increase with higher levels of $\alpha$. Figure $2 b$, in turn, shows the effect on optimal donations $\left(x^{*}\right)$ and disclosure $\left(y^{*}\right)$ of changes in the donor's moneyretention preferences $(\beta)$. The figure shows that both optimal donations and disclosures increase sharply at low levels of donor preferences for retaining money. Both of these results are consistent with expectations from the theoretical economics literature. They are, however, novel within the context of communication research.

Figure $2 \mathrm{c}$ shows the sensitivity of optimal donations $\left(x^{*}\right)$ to organizational efficiency $(\lambda)$, with all other parameters set equal to 1 . As expected, donations increase with the efficiency of the organization. These results are consistent with the empirical research on charitable giving, which has generally found a strong positive linear association between efficiency 
(a)

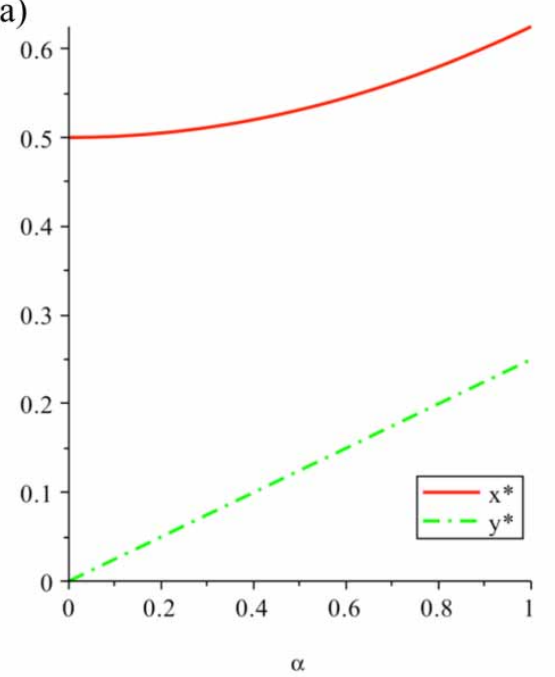

(c)

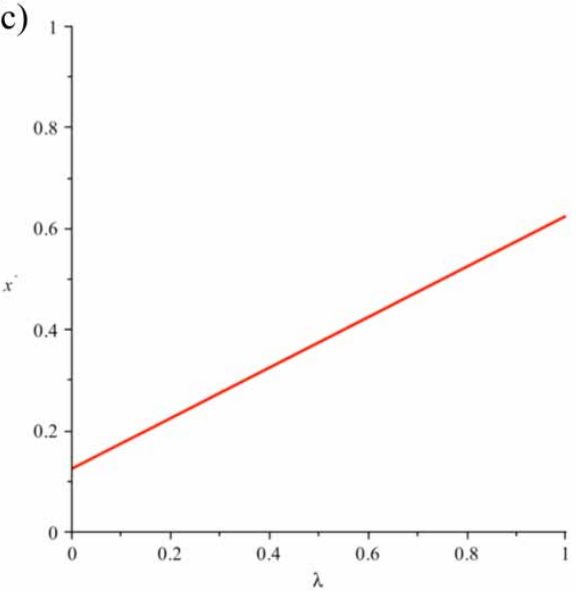

(b)

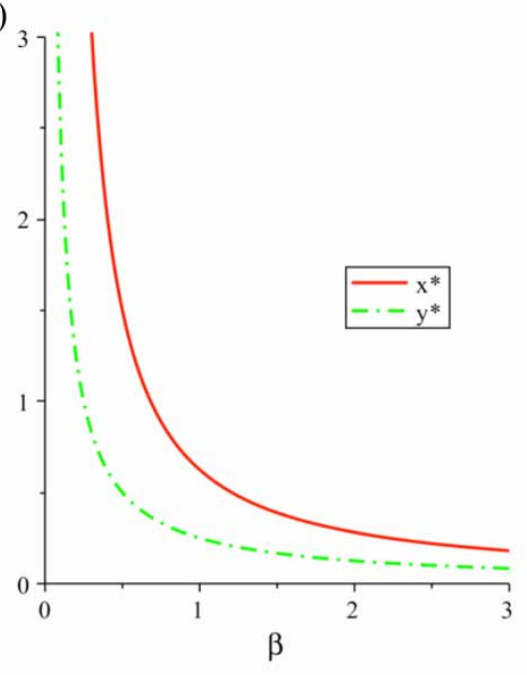

(d)

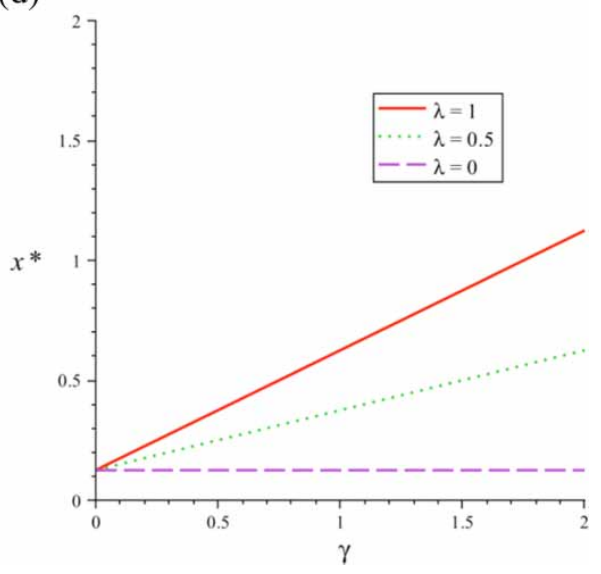

Figure 2. Optimal donations $\left(x^{*}\right)$ and disclosure $\left(y^{*}\right)$ as functions of organizational efficiency $(\lambda)$ and donor's preferences for impact $(\gamma)$, publicity $(\alpha)$, and retaining money $(\beta)$. (a) Optimal donations $\left(x^{*}\right)$ and disclosure $\left(y^{*}\right)$ as functions of donor's publicity preference $(\alpha)$, with $\beta=\lambda=$ $\gamma=1$. (b) Optimal donations $\left(x^{*}\right)$ and disclosure $\left(y^{*}\right)$ as functions of donor's money-retention preference $(\beta)$, with $\alpha=\lambda=\gamma=1$. (c) Optimal donations $\left(x^{*}\right)$ as a function of organizational efficiency $(\lambda)$, with $\alpha=\beta=\gamma=1$. (d) Optimal donations ( $\left.x^{*}\right)$ as a function of donor's efficiency preference $(\gamma)$, for various levels of organizational efficiency $(\lambda)$, with $\alpha=\beta=1$.

ratios and aggregate, annual levels of charitable contributions (e.g., Jacobs \& Marudas, 2009; Tinkelman, 1999). This provides a degree of empirical validation of our model.

More importantly, as shown in Figure $2 \mathrm{~d}$, our findings go beyond existing empirical research to provide novel insights into the disclosure-donations nexus. Figure $2 \mathrm{~d}$ shows the sensitivity of optimal donations $\left(x^{*}\right)$ to the donor's preference for impact $(\gamma)$ at three different levels of organizational efficiency $(\lambda)$. The three lines show a clear monotonic relationship between a donor's impact preference and the 
amount of donations he or she ultimately gives. However, we see important differences in this relationship at various levels of organizational efficiency $(\lambda)$. For a hypothetical fully efficient organization $(\lambda=1)$, the rate of increase in donations as a function of $\gamma$ is higher than for less efficient organizations $(\lambda=0.5)$. At the other extreme, where $\lambda=0$, the donor's preference for maximizing impact $(\gamma)$ does not come into play at all, with optimal donations $\left(x^{*}\right)$ being a horizontal line for all levels of $\gamma$.

These findings may help explain the apparent paradox of how even highly inefficient organizations continue to receive donations. In general, "inefficient" nonprofits are considered to be those below the $75 \%$ efficiency level, and relief efforts often generate "scam" charities that have as little as $0 \%$ of donations going toward spending on programs. Our model predicts, not surprisingly, that such organizations will receive fewer donations. However, as shown in Figure 2d, our model suggests all types of donors appear willing to give - at least a small amount - to charities that are highly inefficient. This holds even for donors with substantial impact-maximizing preferences $(\gamma)$. The model also shows that, for donors with the lowest impact preferences, there is very little difference in the amount of donations given to organizations with different levels of organizational efficiency. The implication is that, instead of basing the donation decision on the level of organizational efficiency, such donors will be driven by other preferences, such as the publicity motive modeled here. Potentially, such donors may be enticed to donate to inefficient charities through the promise of extensive publicity-related disclosures.

Collectively, these are fresh insights into disclosure-donations interactions. We should note that these arguments are at the micro level, at the level of the individual donor. The theoretical insights generated at this level are one of the model's key contributions to the literature. At the same time, we can aggregate these insights to generate hypotheses at the aggregate level, which will allow us to make comparisons with the existing "annual contributions" literature that predominates in accounting and nonprofit studies. For instance, if we were to take three hypothetical organizations, with efficiency ratios of $0 \%, 50 \%$, and $100 \%$, respectively, we would essentially have the three lines in Figure $2 \mathrm{~d}$ as the representation of the relationship between donors' impact preferences and levels of donations. Seen in this light, there is an important theoretical and methodological implication: The slopes of the three lines are different. Thus, in theoretical terms, the relationship between impact preferences and donations is conditional on the level of organizational efficiency; in methodological terms, this suggests the need for a statistical model that can account for the different slope each organization might have. Specifically, this suggests a fixed-effects regression model that includes dummy variables that allow the slope of the impact-preference coefficient to vary according to the organization's level of efficiency (for an overview of such an approach, see Gujarati, 2003). Empirical work should explicitly test for this differential slope hypothesis. 
Figure $2 \mathrm{~d}$ suggests other theoretical implications. First, so long as an organization is minimally efficient, it should target donors with higher impact-maximizing preferences. The figure shows, for example, that a donor with an impact preference $(\gamma)$ of 1.0 will give just as much money to an organization with an efficiency ratio $(\lambda)$ of $50 \%$ as will a donor with an impact preference of 0.5 to an organization with an efficiency ratio of $100 \%$. Overall, the higher the $\gamma$, the higher the donation. Our findings suggest that, controlling for the donor's publicity preference, the level of impact preference $(\gamma)$ is highly consequential. A key implication is that, though organizations should ideally be more concerned with boosting their efficiency and effectiveness, they need also to be concerned with the type of donor they are trying to attract.

\section{Analysis of "Pure-Type" Donors: Sensitivity Analyses for $\gamma+\alpha=1$}

This last insight suggests that, from the organization's perspective, there are two key "ideal types" of donors: the impact-maximizer and the publicity-maximizer. To further explore this intuition, we next conduct a series of analyses with these two hypothetical pure-type donors placed at opposite ends of a continuum. As argued by Doty and Glick (1994), such use of ideal types can be a powerful way of building theory.

The donor's utility function (Equation 2) includes three terms that relate to the donor's preferences for, respectively, impact (associated with $\gamma$ ), personal publicity (associated with $\alpha$ ), and personal cost (associated with $\beta$ ). From the organization's perspective, the first two coefficients $(\gamma$ and $\alpha$ ) are most important, insofar as the organization itself is able to control its level of efficiency as well as the amount of publicity it affords its donors. Accordingly, let us now consider the case where, analogous to what we see in a Cobb-Douglas production function with constant returns to scale, donors' preferences for maximizing efficiency and publicity sum up to 1 ; i.e., we consider the case where $\gamma+\alpha=1$. All of the sensitivity analyses that follow reflect this condition, such that a value of $\alpha=1$ indicates a pure "publicitymaximizing" donor and $\alpha=0$ indicates a pure "impact-maximizing" donor.

Figure 3a shows the sensitivity of optimal donations $\left(x^{*}\right)$ to donors' publicity preferences $(\alpha)$ at three different levels of organizational efficiency $(\lambda)$, with the other parameters set equal to 1 . We see that a pure publicity maximizer $(\alpha=1)$ will donate the same amount regardless of the organization's level of efficiency. Such is not the case for impact maximizers. At the lowest level of efficiency $(\lambda=0)$, a pure impact maximizer $(\alpha=0)$ will not donate. However, at middle and high levels of efficiency, a pure impact-maximizer will donate more than a pure publicity-maximizer. The figure also shows the organization's gain in donations from greater efficiency increases dramatically as $\alpha$ decreases. These are novel findings worth testing in an empirical setting.

Figure $3 b$, in turn, shows the sensitivity of optimal donations $\left(x^{*}\right)$ to organizational efficiency $(\lambda)$ at different levels of donor preferences for publicity $(\alpha)$. The results in this figure carry several interesting empirical implications. With an efficiency $(\lambda)$ 
(a)

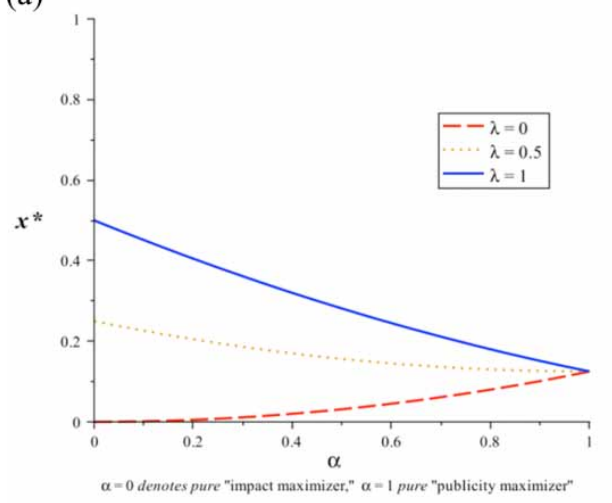

(c)

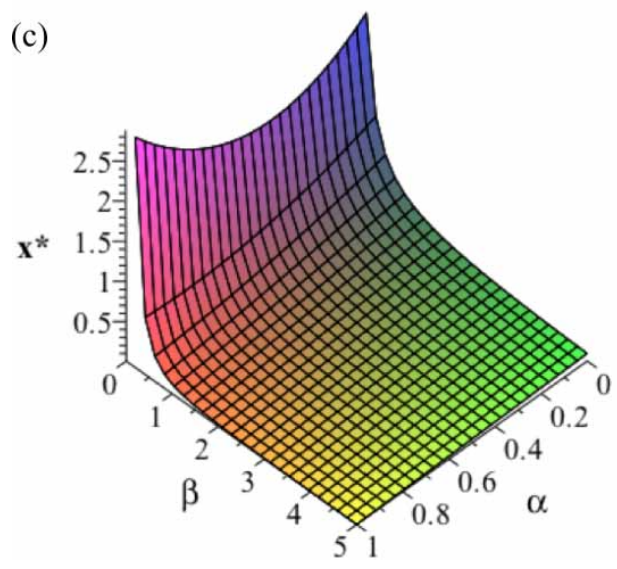

(b)

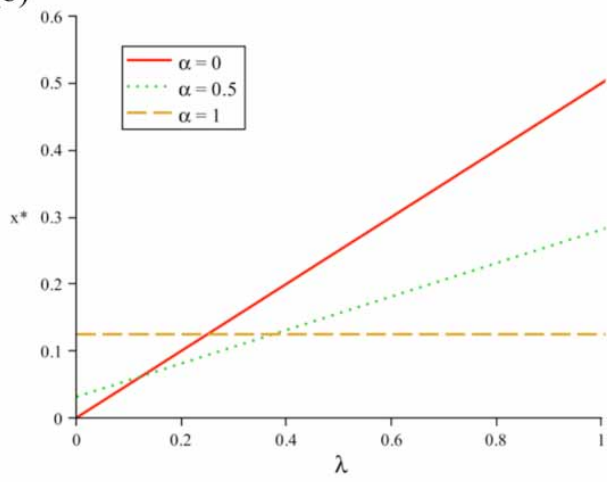

Figure 3. Sensitivity of optimal donations $\left(x^{*}\right)$ to organizational efficiency $(\lambda)$ and donor's preference for publicity $(\alpha)$ and money retention $(\beta)$, where $\gamma+\alpha=1 \quad(\alpha=0$ denotes pure "impact maximizer," $\alpha=1$ denotes pure "publicity maximizer"). (a) Sensitivity of optimal donations $\left(x^{*}\right)$ to donor's publicity preference $(\alpha)$ at different levels of organizational efficiency $(\lambda)$, with $\beta=1$. (b) Sensitivity of optimal donations $\left(x^{*}\right)$ to organizational efficiency $(\lambda)$ at different levels of donor's publicity preference $(\alpha)$, with $\beta=1$. (c) Optimal donations $\left(x^{*}\right)$ as a function of donor's preference for publicity $(\alpha)$ and money retention $(\beta)$, where $\gamma+\alpha=1$, with $\lambda=1$.

value of 0 , indicating a likely scam organization, a pure impact-maximizing donorone who cares only about the effectiveness of the programs delivered by the organization - will not make a donation. However, as $\alpha$ increases to any level above zero, indicating he or she has at least minimal preferences for personal publicity, the level of donations will be some positive amount. At the extreme, the figure shows that the pure publicity-maximizing donor $(\alpha=1)$ does not care about the level of efficiency of the organization-donations are the same regardless of the program spending ratio. These findings may partially explain the persistence of both "scam" organizations and highly inefficient organizations. So long as an organization can utilize disclosure to publicize key donors' contributions, especially those with high 
publicity-preference coefficients, the organization can mitigate the negative effects of low levels of organizational efficiency. Given the social costs resulting from both fraudulent and highly inefficient organizations, this is something that deserves further empirical investigation.

Seen from a different vantage point, let us consider that organizations at the lower range of organizational efficiency, say $\lambda \leq 0.2$, are "highly inefficient" organizations, that those in the $0.2<\lambda<0.7$ range are "moderately inefficient" organizations, and that those with $\lambda>0.8$ are "highly efficient" organizations. Figure $3 \mathrm{~b}$ suggests there are starkly different payoffs from targeting particular types of donors for these three types of organizations. "Highly inefficient" (or worse, scam) organizations have an incentive to target the pure publicity-maximizers. In contrast, for the "moderately inefficient" organizations in the middle of the $\mathrm{x}$-axis (e.g., Kiwanis International, with $\lambda=0.42$ ), there are not large differences in the donation given by a pure publicity maximizer $(\alpha=1)$ or a donor with mixed preferences $(\alpha=0.5)$, with a somewhat larger amount granted by a pure impact maximizer $(\alpha=0)$. This suggests it is not as critical for these organizations to carefully target a specific type of donor. Such is not the case for "highly efficient" organizations such as Americares $(\lambda=$ 0.99): As can be seen in the considerable differences in donation amount given by donors with varying levels of $\alpha$, targeting the "right" type of donor is a critical organizational decision. Such organizations would generally benefit from courting impact rather than publicity maximizers.

Last, Figure $3 c$ shows the sensitivity of optimal donations $\left(x^{*}\right)$ to donors' publicityseeking $(\alpha)$ and money-retention $(\beta)$ preferences, with organizational efficiency $(\lambda)$ set equal to 1 . One interesting feature that this three-dimensional graph shows, where $\alpha=1$ indicates a pure publicity-maximizer and $\alpha=0$ indicates a pure impactmaximizer, is that donations are higher for the "pure" types of donors than they are for donors with "mixed" preferences for impact and publicity. Organizations may thus be able to maximize donations by implementing substantial disclosure policies targeted at donors with strong publicity preferences while striving to boost efficiency and effectiveness for the impact-maximizing donors.

\section{Discussion and Conclusions}

In formally modeling the strategic and interactive nature of organizational communication, our model clarifies the nature of disclosure-donation interactions in a way that carries theoretical and practical insights for organizations, donors, and communication scholars alike. This parsimonious, yet informative, model considers three core donor preferences: impact, publicity, and cost. Prior game-theoretic models have not accounted for the desire for impact, and the empirical literature has generally not considered the desire for publicity. Our model makes a strong case for considering the joint impact of these key preferences in future research.

Communication scholars would in particular benefit from greater use of formal theories of the interconnectedness of donor preferences and organizational transparency. What types of insight might we gain from such an approach? For 
one thing, our model suggests that, when it comes to disclosure, more is not necessarily better. Though there is a strong positive association between disclosure and donations, it is not a one-to-one relationship. There is instead an "optimal level" of disclosure that depends on the interrelated decisions made by the producer and the consumer of the information.

The study effectively delivers three levels of theoretical insights: (1) findings that are expected and conform with existing literature but are extended by our analyses; (2) findings that are not unexpected, but are not found in the current literature; and 3) novel insights, those that are unexpected and not in the current literature. In the first category are such findings as a linear relationship between organizational efficiency and equilibrium levels of donations. In the second category are such findings as the importance of publicity-seeking preferences in determining donation amounts - something not discussed in the communication literature-and the finding that equilibrium levels of disclosure are not sensitive to levels of organizational efficiency. And in the third category are such findings as a curvilinear relationship between publicity preferences and donations, a relationship between impact preferences and donations that is conditional on the level of organizational efficiency, and various other insights that flow from the "ideal type" analyses that focused on two pure-type donors: impact maximizers and publicity maximizers.

Our study thus reflects the theory-building potential of ideal-type analyses (Doty \& Glick, 1994). Through such analysis, we are able to further specify the relationship between donors' desire for impact and donations. Prior empirical literature, employing annually aggregated donations data (see Parsons, 2003, for a review), has posited a blunt relationship equivalent to "donations are positively related to organizational efficiency." Our model clarifies the nature of this relationship in at least two key ways. First, it goes beyond existing empirical research by adding a micro-level theoretical explanation for the donor behavior seen in the empirical literature's annually aggregated relationships; our model effectively suggests the theoretical intuition at the individual level. Second, our model posits a more precise relationship: Donations depend both on the type of donor and the type of organization; differently put, the variables are related, but they are related differently for different types of organizations and different types of donors. By aggregating, the empirical regression-based studies are "washing away" all the nuance. And it is precisely this type of nuance that our game-theoretic model provides.

We believe the approach taken in this paper would yield benefits for interpersonal and organizational communication scholars interested in a wide range of phenomena related to prosocial behavior, individual and organizational disclosure, attitudebehavior relationships, or the effects of institutional messages. The fact is, decision making is important in many areas of communication. Typically, these domains have multiple decision-makers - with a mix of common and conflicting objectives - whose individual decisions impact the others' payoffs. Game-theoretic applications are ideally designed to examine such decisions, whether they occur in fundraising campaigns, political campaigns, marketing campaigns, online dating, public relations, negotiations, workplace transparency, crisis communications, or doctor-patient 
interactions. We thus encourage communication scholars to take up the call and develop their own formal models that extend, translate, and challenge our findings.

\section{Practical Applications}

In addition to being a descriptive theory, game theory is a normative theory that is often used as a guide in decision making even before empirical validation. For instance, purely theoretical (nontested) game-theoretic models were used to design US government auctions that sold $\$ 42$ billion worth of access to the wireless spectrum from 1994 to 2001 (McMillan, 2002). Similarly, Axelrod's groundbreaking 1984 book The Evolution of Cooperation has engendered numerous practical decisionmaking insights even without "real" data.

Our model likewise carries normative implications. Some of the most salient applications derive from our theoretical emphasis on two ideal-type donors: the impact maximizer and the publicity maximizer. When seen through the prism of two ideal-type donors, the manager has a guide for strategically thinking through communicative decision making. Specifically, our model implies that, after assessing the organization's own strengths and weaknesses in the areas of disclosure and efficiency, the manager should undertake a stakeholder mapping ( $\mathrm{Kim}, \mathrm{Ni}$, \& Sha, 2008) of donor groups according to their preferences for efficiency and publicity. The reason, as highlighted by our model, is that there are different payoffs to the organization depending on the preferences of the donor and the characteristics of the organization.

As suggested earlier in studying Figure 3b, we can think of there being three categories or "types" of organizations - highly inefficient, moderately inefficient, and highly efficient - that see substantially different payoffs according to whom they target in their fundraising activities. Notably, "highly inefficient" and scam organizations $(\lambda \leq 0.2)$ have a strong incentive to target pure publicity-maximizers, "moderately inefficient" organizations $(0.2<\lambda<0.7)$ will do fine targeting any type of donor, and "highly efficient" organizations $(\lambda>0.8)$ have a strong incentive to target impact maximizers. The lesson: Targeting the "right" donor for your organization is a critical managerial decision.

In short, our model clarifies the nature of disclosure-donation interactions. Organizations now have a framework for matching their organizational type (low, medium, or high efficiency) with the type of donor (impact-maximizer or publicitymaximizer) they want to or should be marketing to. Through this framework, moreover, organizations can gain a sense of the tradeoffs involved with different strategic communication choices.

Our model thus provides insights into new ways for organizations to approach decision making and their relationships with different categories of donors. Though we do not know the exact proportions of donors who are publicity-seekers, there is ample evidence that many donors do want publicity - examples highlighting such demand include the growing number of social media-based crowdfunding sites such as Kickstarter, GoFundMe, or Kiva, where each donation is publicized and few donors 
choose to be anonymous. And from the empirical accounting and nonprofit studies literatures there is strong evidence of donors' desire to have an impact. Our model provides practical insights into organizations' efforts to communicate with such donors.

The game-theoretic approach used here is thus highly useful for capturing the basic forces at play in any given strategic situation. In forcing the manager to "visualize and anticipate" the other actors' moves, it also facilitates cooperation, inasmuch as the manager employing the model will better understand stakeholders' motivations. In short, the model advances general principles (Dixit et al., 2009) that foster learning around why things happen and provide "a ready reference point" (p. 5) for application in new areas of organization-stakeholder interaction.

\section{Example: Applications for Crowdfunding Sites}

To shed light on some of the most salient practical applications, we will briefly explore the actionable ramifications for crowdfunding sites such as GoFundMe (www.gofundme. com), Crowdrise (www.crowdrise.com), Kickstarter (www.kickstarter.com), and Facebook Causes, among others. In many respects, such sites are practically designed for the publicity-seeking donor - they are often plugged into Facebook, which allows donors' "friends" to see the donation activity in their feeds. Salient design features highlight recent donations, "friends" of the cause/organization, and other such publicity and social features. As a result, such sites seem to be made with the social publicity-seeker in mind - they typically have little "impact" information. As a result, the designers of such systems may (inadvertently or not) be working against the interests of donors with no publicity-seeking preferences. Moreover, they better serve the purposes of "splashy," publicity-yielding projects and causes as opposed to lessexciting, more long-term but potentially more impactful programs and charities.

One product of the rapid, publicity-seeking crowdfunding model is the possibility of scams, such as those that have recently affected Kickstarter. Notably, our model would predict such scams in identifying conditions where publicity-seeking donors are susceptible to malfeasance. The model also leads to the conclusion that organizations and regulators alike need to develop mechanisms to avert the possibility of widespread scams.

Building on our earlier recommendations about considering the interests of two ideal-type donors, it becomes apparent that these crowdfunding sites are not serving the interests of impact-maximizing donors. Such donors have the choice only of receiving a public acknowledgement or of making the donation anonymously. This opt-out approach to fundraising disclosure is better than nothing, but it does not help impact-maximizing donors learn what they are most interested in: The extent to which their donation will change lives for the better. Crowdfunding sites should therefore seek to overcome the dearth of impact and efficiency information that is currently available on these sites.

We should note that a manager employing our model as a decision-making tool would only exclude such donors by design; otherwise, the manager would map the interests of both groups, and see that the site is not meeting the interests of impact- 
maximizing donors. The model also serves to sensitize donors on these sites to the possibility of "cheap talk" and manipulative fundraising disclosure. For the donor, insights derive from asking "What is most important to me?," studying the disclosure and efficiency characteristics of a set of nonprofits, and then basing donation decisions on the resultant analyses. By viewing donation-disclosure interactions as a game of strategy, donors stand a better chance of greater satisfaction, of finding synergy with like-minded organizations, and of being safeguarded against potential manipulation.

More generally, organizations - and organizational communication scholarsneed to explicitly consider the characteristics of the recipients of organizational messages, and how these characteristics in turn influence organizational decision making. This is an aspect that has been generally underexamined in the communication literature. Though organizational communication scholars have brought us far, the inclusion of strategic decision-making and target audience preferences in the literature would deliver even further insights into the determinants and outcomes of organizational disclosure.

\section{Acknowledgements}

The authors would like to thank Tom Feeley, Frank Tutzauer, Ashley Anker, Gina Reinhardt, Frank Zagare, and Phil Arena for helpful comments and suggestions. We would also like to acknowledge the valuable feedback we received from participants at the 2012 annual meeting of the International Communication Association in Phoenix, AZ. This research was partially supported by the National Science Foundation under award number 1200899, and US Department of Homeland Security through the National Center for Risk and Economic Analysis of Terrorism Events (CREATE) under award number 2010-ST-061-RE0001. However, any opinions, findings, conclusions, or recommendations in this document are those of the authors and do not necessarily reflect views of the sponsors.

\section{References}

Anker, A. E., Feeley, T. H., \& Kim, H. (2010). Examining the attitude-behavior relationship in prosocial donation domains. Journal of Applied Social Psychology, 40, 1293-1324.

Arpan, L. M., \& Roskos-Ewoldsen, D. R. (2005). Stealing thunder: Analysis of the effects of proactive disclosure of crisis information. Public Relations Review, 31, 425-433.

Axelrod, R. (1984). The evolution of cooperation. New York, NY: Basic Books.

Behn, R. D. (2005). The accountability dilemma. Public Management Report, 2, 1-2.

Bergstrom, T., Blume, L., \& Varian, H. (1986). On the private provision of public goods. Journal of Public Economics, 29, 25-29.

Bishop, C. (2006, June 16). The internationalization of secrecy: A look at transparency within the World Trade Organization. Paper presented at the annual meeting of the International Communication Association, Dresden, Germany.

Blackwood, A., Wing, K., \& Pollak, T. H. (2008). The nonprofit sector in brief: Facts and figures from the Nonprofit Almanac 2008. Washington, DC: Urban Institute.

Carpenter, J., Connolly, C., \& Myers, C. (2008). Altruistic behavior in a representative dictator experiment. Experimental Economics, 11, 282-298. 
Clark, J. (2002). Recognizing large donations to public goods: An experimental test. Managerial and Decision Economics, 23, 33-44.

Dixit, A., Skeath, S., \& Reiley, D. (2009). Games of strategy (3rd ed). New York, NY: Norton.

Doty, D., \& Glick, W. (1994). Typologies as a unique form of theory building: Toward improved understanding and modeling. Academy of Management Review, 19, 230-251.

Fiacco, A. V., \& Ishizuka, Y. (1990). Sensitivity and stability analysis for nonlinear programming. Annals of Operations Research, 27, 215-235.

Fink, E. L. (1993). Mathematical models for communication: An introduction. Journal of Communication, 43, 4-7.

Gandía, J. L. (2011). Internet disclosure by nonprofit organizations: Empirical evidence of nongovernmental organizations for development in Spain. Nonprofit and Voluntary Sector Quarterly, 40, 57-78.

Gordon, T. P., Knock, C., \& Neely, D. G. (2009). The role of rating agencies in the market for charitable contributions: An empirical test. Journal of Accounting and Public Policy, 28, 469484 .

Gujarati, D. (2003). Basic econometrics (4th ed). New York, NY: McGraw-Hill.

Hancock, J. T., \& Toma, C. L. (2009). Putting your best face forward: The accuracy of online dating photographs. Journal of Communication, 59, 367-386.

Healy, P. M., \& Palepu, K. G. (2001). Information asymmetry, corporate disclosure, and the capital markets: A review of the empirical disclosure literature. Journal of Accounting and Economics, $31,405-440$.

Huang, Y. (2001). OPRA: A cross-cultural, multiple-item scale for measuring organization-public relationships. Journal of Public Relations Research, 13, 61-90.

Jacobs, F. A., \& Marudas, N. P. (2009). The combined effect of donation price and administrative inefficiency on donations to US nonprofit organizations. Financial Accountability and Management, 25, 33-53.

Kent, M. L., Taylor, M., \& White, W. J. (2003). The relationship between web site design and organizational responsiveness to stakeholders. Public Relations Review, 29, 63-77.

Kim, J., Ni, L., \& Sha, B. (2008). Breaking down the stakeholder environment: Explicating approaches to the segmentation of publics for public relations research. Journalism and Mass Communication Quarterly, 85, 751-768.

Lewis, L. (2005). The civil society sector: A review of critical issues and research agenda for organizational communication scholars. Management Communication Quarterly, 19, 238267.

Lovejoy, K., \& Saxton, G. D. (2012). Information, community, and action: How nonprofit organizations use social media. Journal of Computer-Mediated Communication, 17, 337-353.

McMillan, J. (2002). Reinventing the bazaar. New York, NY: Norton.

Moore, M. (2000). Managing for value: Organizational strategy in for-profit, nonprofit, and governmental organizations. Nonprofit and Voluntary Sector Quarterly, 29, 183-208.

Morrow, J. (1994). Game theory for political scientists. Princeton, NJ: Princeton University Press.

Myers, S. A., \& Johnson, A. D. (2004). Perceived solidarity, self-disclosure, and trust in organizational peer relationships. Communication Research Reports, 21, 75-83.

Parsons, L. M. (2003). Is accounting information from nonprofit organizations useful to donors? A review of charitable giving and value relevance. Journal of Accounting Literature, 22, 104-129.

Rawlins, B. (2009). Give the emperor a mirror: Toward developing a stakeholder measurement of organizational transparency. Journal of Public Relations Research, 21, 71-99.

Reck, J. (2001). The usefulness of financial and nonfinancial performance information in resource allocation decisions. Journal of Accounting and Public Policy, 20, 45-71.

Reinhardt, G. Y. (2009). Matching donors and nonprofits: The importance of signaling in funding awards. Journal of Theoretical Politics, 21, 283-309.

Romano, R., \& Yildirim, H. (2001). Why charities announce donations: A positive perspective. Journal of Public Economics, 81, 423-447. 
Rosenfeld, L. B., Richman, J. M., \& May, S. K. (2004). Information adequacy, job satisfaction and organizational culture in a dispersed-network organization. Journal of Applied Communication Research, 32, 28-54.

Sargeant, A. (2001). Relationship fundraising: How to keep donors loyal. Nonprofit Management and Leadership, 12, 177-192.

Sargeant, A., Ford, J., \& West, D. (2006). Perceptual determinants of nonprofit giving behavior. Journal of Business Research, 59, 155-165.

Saxton, G. D., \& Guo, C. (2011). Accountability online: Understanding the Web-based accountability practices of nonprofit organizations. Nonprofit and Voluntary Sector Quarterly, 40, 270-295.

Saxton, G. D., Neely, D. J., \& Guo, C. (2009). Web disclosure and the market for charitable contributions. Paper presented at the annual meeting of the American Accounting Association, New York, NY, August 1-5, 2009.

Stefanone, M. A., \& Jang, C.-Y. (2008). Writing for friends and family: The interpersonal nature of blogs. Journal of Computer-Mediated Communication, 13, 123-140.

Strom, S. (2011). A charitable rush, with little direction. New York Times, March 16, p. A14.

Sweetser, K. D. (2010). A losing strategy: The impact of nondisclosure in social media on relationships. Journal of Public Relations Research, 22, 288-312.

Tinkelman, D. (1999). Factors affecting the relation between donations to not-for-profit organizations and an efficiency ratio. Research in Government and Nonprofit Accounting, $10,135-161$.

Weisbrod, B. A., \& Dominguez, N. D. (1986). Demand for collective goods in private nonprofit markets: Can fundraising expenditures help overcome free-rider behavior? Journal of Public Economics, 30, 83-96.

Zagare, F. (1984). Quantitative applications in the social sciences: Vol. 41. Game theory: Concepts and applications. Newbury Park, CA: Sage.

Zhuang, J., \& Bier, V. (2010). Reasons for secrecy and deception in homeland-security resource allocation. Risk Analysis, 30, 1737-1743.

Zhuang, J., \& Bier, V. (2011). Secrecy and deception at equilibrium, with applications to antiterrorism resource allocation. Defence and Peace Economics, 22, 43-61.

\section{Appendix: Proof of Equilibrium Solution}

With sequential-move games, the solution is based on the concept of subgame perfect Nash equilibrium (SPNE), a subset of the classic Nash equilibrium concept. SPNE solutions are found using the method of backward induction (Dixit et al., 2009). Here we provide the solution for $\alpha \geq 0$. There is also a solution for $\alpha<0$ that is less interesting from a theoretical perspective and is thus not shown (available upon request).

\section{Definition 1}

We call a pair $\left(x^{*}, y^{*}\right)$ a subgame perfect Nash equilibrium (SPNE) for our model if and only if the following two conditions hold:

$$
\begin{gathered}
x^{*}=\hat{x}\left(y^{*}\right)=\underset{x}{\arg \max } U_{D}(x, y) \\
y^{*}=\underset{y}{\arg \max } U_{N}(\hat{x}(y), y)
\end{gathered}
$$




\section{Solution}

We can now solve for the equilibrium $\left(x^{*}, y^{*}\right)$ as defined in Definition 1 . The donor moves second, so we first take the derivative of the donor's maximization problem as shown earlier, $U_{D}(x, y)=\gamma \lambda x+\alpha x y-\beta x^{2}$, and solve for the donor's best response, $\hat{x}(y)$ :

$$
\frac{d U_{D}(x, y)}{d x}=\gamma \lambda+\alpha y-2 \beta x=0 \Rightarrow \hat{x}(y)=\frac{\gamma \lambda+\alpha y}{2 \beta}
$$

where the second order condition holds because $\frac{d^{2} U_{D}(x, y)}{d x^{2}}=-2 \beta<0$. Now, we substitute the donor's best response function $\hat{x}(y)$ into the organization's (first-mover's) utility function:

$$
U_{N}(\hat{x}(y), y)=\frac{\gamma \lambda+\alpha y}{2 \beta}-y^{2}
$$

Differentiation provides

$$
\frac{d U_{N}}{d y}=\frac{\alpha}{2 \beta}-2 y=0 \Rightarrow y^{*}=\frac{\alpha}{4 \beta}
$$

where the second order condition holds because $\frac{d^{2} U_{N}(x, y)}{d y^{2}}=-2<0$. We then substitute this value for $y^{*}$ into the donor's best response function $\hat{x}(y)$ as noted earlier:

$$
x^{*}=\hat{x}\left(y^{*}\right)=\frac{\gamma \lambda+\alpha y^{*}}{2 \beta}=\frac{\gamma \lambda+\alpha\left(\frac{\alpha}{4 \beta}\right)}{2 \beta}=\frac{4 \beta \gamma \lambda+\alpha^{2}}{8 \beta^{2}}
$$

In summary, the subgame perfect Nash equilibrium is the following:

$$
\left(x^{*}, y^{*}\right)=\left(\frac{4 \beta \gamma \lambda+\alpha^{2}}{8 \beta^{2}}, \frac{\alpha}{4 \beta}\right)
$$

